Report no. $97 / 11$

\title{
On adjoint equations for error analysis and optimal grid adaptation in CFD
}

\author{
M. B. Giles
}

This paper explains how the solutions of appropriate adjoint equations can be used to estimate the errors in important integral quantities, such as lift and drag, obtained from CFD computations. These error estimates can be used to obtain improved estimates of the integral quantities, or as the basis for optimal grid adaptation.

The theory is presented for both finite volume and finite element approximations. For a node-based finite volume discretisation of the Euler equations on unstructured grids, the adjoint analysis makes it possible to prove second order accuracy. A superconvergence property is proved for a finite element discretisation of the Laplace equation, and references are provided for the extension of the analysis to the convection/diffusion and incompressible Navier-Stokes equations.

This paper was presented at the symposium Computing the Future II: Advances and Prospects in Computational Aerodynamics to honour the contributions of Prof. Earll Murman to CFD and the aerospace community.

Key words and phrases: error analysis, adjoint equations, grid adaptation

This research was supported by EPSRC research grant GR/K91149.

Oxford University Computing Laboratory

Numerical Analysis Group

Wolfson Building

Parks Road

Oxford, England OX1 3QD

http: //www.comlab.ox.ac.uk

email: giles@comlab.oxford.ac.uk

September, 1997 


\section{Introduction}

One challenge facing CFD is to be able to give tight error bounds so that an engineer knows the accuracy of the computed results. Leaving aside the difficult issue of assessing the magnitude of modelling errors due to turbulence and transition prediction, this requires an accurate estimate of the errors due to the discretisation of the system of p.d.e.'s. With such knowledge, one can then hope to develop a rigorous approach to optimal grid adaptation, to produce the most accurate solution for a given computational cost, or to minimise the computational cost in achieving a given level of accuracy.

At present, there is still a considerable gap between mathematical theory and engineering practice. When using smooth structured grids for smooth flow fields with no singularities, the order of accuracy can be deduced from an analysis of the truncation error. The absolute magnitude of the error for a particular grid size can be estimated from past experience with grid refinement studies on test problems. However, when one starts using grid redistribution (moving grid points) to improve the resolution of flow features, the grid is no longer smooth, and if one uses local grid refinement (adding additional grid points) the grid becomes unstructured, at least from the point of view of theoretical analysis if not from the programming perspective.

For unstructured grids the current practice in grid refinement remains the use of heuristic methods. Some of these are based on well-founded physical reasoning, that one needs to have good resolution of features such as shocks, boundary layers, wakes and free shear layers. However, it is entirely possible that too much computational effort is put into the resolution of these features at the expense of insufficient resolution of other parts of the flow field such as the smooth but rapid expansion over the leading edge of an airfoil. Other methods are based on the idea of reducing the magnitude of the truncation error, but this takes no account of the magnitude of the solution error caused by that truncation error.

Until recently, the rigorous mathematical approach to error analysis for unstructured grids has involved the use of the Aubin-Nitsche technique to derive error bounds for finite element approximations of model problems such as the convection-diffusion equation [1]. However, when applied to approximations of hyperbolic p.d.e.'s this usually results in error bounds using negative Sobolev norms (e.g. [2]) which have little engineering significance. Therefore, this has generally been of little help to engineers although it has led to practical grid refinement indicators $[3,4]$.

Very recently, however, a promising new approach to error analysis and optimal grid refinement has been introduced by Becker \& Rannacher, Süli \& Giles, and Paraschivoiu, Patera and Peraire. This starts from the observation that in many cases the key quantities of engineering interest are functionals of the solution, such as the lift and drag on an airfoil. Therefore, the most relevant 
measure of the solution error is the absolute error in these derived quantities. This leads to a mathematical analysis involving the adjoint p.d.e. with inhomogeneous terms and boundary conditions appropriate to the particular functional. The resulting adjoint solution defines the relationship between the error in the functional and the finite element residual error, which is the extent to which the finite element solution is not the solution of the original analytic problem. Thus, an estimate of the adjoint solution together with the local finite element residual error can be used to define an optimal grid refinement strategy to obtain the most accurate prediction of lift and drag for a given computational cost.

This line of research is still in its infancy. Drawing on theory developed for elliptic p.d.e.'s in structural analysis $[5,6,7]$, Becker and Rannacher developed a posteriori error estimates for the incompressible Navier-Stokes equations [8]. In addition to similar a posteriori error estimates, Süli, Giles et al have also developed a priori error estimates for the incompressible Navier-Stokes equations, proving an interesting superconvergence property [9]. Paraschivoiu, Patera and Peraire have also developed a slightly different analysis employing adjoint solutions to obtain upper and lower bounds for functionals for elliptic p.d.e.'s [10] with the aim of proceeding to the Navier-Stokes equations.

The aim of this paper is to explain this approach to error analysis and show how it can be used for optimal grid adaptation. The first section outlines an a priori error analysis for finite volume discretisations of the Euler equations on both structured and unstructured grids. The error estimates can be used either to improve the computed value for the functional, or as the basis for grid adaptation through redistribution or refinement. In addition, it is shown that for unstructured grids the use of a conservative discretisation ensures that the order of accuracy of the functional is one greater than the order of the truncation error of the finite volume discretisation.

The second section presents the theory for a finite element discretisation of a simple elliptic model problem, discussing the superconvergence property arising from the a priori error analysis, and the use of the a posteriori error analysis for grid adaptation. This provides an introduction to the literature on finite element error analysis; references are provided for the extension of the analysis to the convection/diffusion and incompressible Navier-Stokes equations.

\section{Finite volume analysis}

The analysis begins with the discrete equations arising from a finite volume approximation of the original fluid dynamic equations,

$$
R^{h}\left(U^{h}\right)=0 .
$$

Here $U^{h}$ is the discrete flow solution and the equations come directly from a flux balance and are not normalised by the area or volume of the computational cells. 
The solution error $e^{h}$ is defined by

$$
U^{h}=U+e^{h},
$$

where $U$ is the analytic flow solution. Linearising the discrete equations gives

$$
R^{h}(U)+\frac{\partial R^{h}}{\partial U^{h}} e^{h} \approx 0
$$

where $R^{h}(U)$ is the vector of truncation errors obtained by substituting the analytic solution into the discrete operator. This equation describes the relationship between the truncation error, which is relatively easy to estimate, and the solution error which is the quantity of greater interest.

If $I(U)$ is the scalar functional of interest (e.g. lift or drag) based on the analytic solution, then the error in the corresponding discrete approximation, $I^{h}\left(U^{h}\right)$, can be broken into two components,

$$
I^{h}\left(U^{h}\right)-I(U)=\left(I^{h}\left(U^{h}\right)-I^{h}(U)\right)+\left(I^{h}(U)-I(U)\right) .
$$

The second term is the truncation error in approximating the operator $I$. The first term is due to the error in the discrete solution $U^{h}$ and can be approximated as follows,

$$
\begin{aligned}
I^{h}\left(U^{h}\right)-I^{h}(U) & \approx \frac{\partial I^{h}}{\partial U^{h}} e^{h} \\
& =-\frac{\partial I^{h}}{\partial U^{h}}\left(\frac{\partial R^{h}}{\partial U^{h}}\right)^{-1} R^{h}(U) \\
& =V^{T} R^{h}(U),
\end{aligned}
$$

where the vector $V$ is the solution of the adjoint flow equations,

$$
\left(\frac{\partial R^{h}}{\partial U^{h}}\right)^{T} V+\left(\frac{\partial I^{h}}{\partial U^{h}}\right)^{T}=0 .
$$

Thus the adjoint flow solution relates the errors in quantities such as lift and drag, to the underlying truncation errors in the evaluation of finite volume cell residuals.

The role of the adjoint flow solution in optimal design is now well established $[11,12,13,14,15]$. The fact that the same adjoint solution plays a critical role in error analysis should not be surprising. In design one is concerned with the perturbation of a functional due to changes in the geometry; in error analysis one is concerned with the perturbation of the functional due to the truncation errors. 


\section{Structured grids}

For structured grids in which one wishes to improve the accuracy through grid redistribution, moving the grid points to better resolve regions of large flow variation, this analysis can be used to define an optimal adaptation strategy.

Consider the discretisation of the Euler equations in $2 \mathrm{D}$ or $3 \mathrm{D}$ on a smooth structured grid. Taylor series expansions can be used to analyse the truncation error of the discretisation. Bearing in mind that the residuals are not normalised by the area or volume of the computational cells the truncation error is of order $h^{p+d}$ where $h$ is the length of the cell (assumed to have a fixed aspect ratio to simplify the analysis), $p$ is the order of accuracy and $d$ is the dimension of the problem. After computing the adjoint solution, and using the flow solution to estimate the higher order derivatives in the truncation error, the overall error in the functional can be expressed as a sum of the following form

$$
\sum_{j} h_{j}^{p+d} T_{j}
$$

Here the index $j$ denotes the individual locations of the flow variables (usually at nodes or cell centres) and $h_{j}$ is the length of the associated cell. $T_{j}$ is a scalar which involves the product of the adjoint solution and higher order derivatives of the flow solution coming from the truncation error estimation. A numerical estimate of the value of these higher order derivatives can be obtained from a computed flow solution by using local least-squares approximation by a high order polynomial. An adjoint flow calculation provides the adjoint solution and hence one can obtain an accurate estimate for the quantity $T_{j}$ at each node.

The resulting error estimate for the functional can be used in two ways. The first is to use it as a correction to the computed value of the functional, providing a more accurate value at the cost of an adjoint flow computation. If one is interested in more than one functional then each would require an adjoint flow computation, but this might still be computationally less expensive than improving the accuracy through using a finer grid for the original flow calculation.

The second option is to use the error estimate for optimal grid adaptation through redistribution, moving grid points to better resolve flow features. The objective would be to minimise

$$
\sum_{j} h_{j}^{p+d}\left|T_{j}\right|
$$

Taking $h_{j}^{d}$ to be the area $(d=2)$ or volume $(d=3)$ of the cell, this summation can be approximated by the integral

$$
\int h^{p}|T| d V
$$


where $|T|$ is a continuous approximation to $\left|T_{j}\right|$. Similarly, the total number of cells is given approximately by

$$
\int h^{-d} d V
$$

Minimising the first integral while keeping the latter fixed, using a Lagrange multiplier, leads to the requirement that $h^{p+d}|T|$ should be uniform.

In practice, one would usually be concerned with more than one functional, such as both lift and drag. To handle this, the adaptation criterion could be amended so that the strategy is to ensure that

$$
h^{p+d} \sum_{m}\left|T^{(m)}\right|
$$

is approximately uniform, where $T^{(m)}$ are the corresponding error components for the functionals of concern. In principle, the construction of each $T^{(m)}$ requires the solution of an adjoint equation. However, in practice it may be that these adjoint solutions can be approximated sufficiently well for adaptation purposes by simple analytic functions, based on a detailed understanding of their origin and qualitative nature [16].

This strategy may not seem very different from current adaptation practices which aim to make the truncation error uniform across the grid. The crucial difference however is the inclusion of the adjoint solution which reflects the fact that not all truncation errors are equal in their effect on the quantities of engineering interest such as lift and drag. A good example of this is truncation errors in the wake behind an airfoil. It is not uncommon for the wake to be poorly resolved a chord or more downstream of an airfoil, due to grid generation difficulties in anticipating the trajectory of the wake. However, although the resulting truncation errors may be relatively large, the adjoint flow solution for lift and drag functionals is relatively small, reflecting the fact that these errors do not significantly affect the flow near the airfoil. Thus, adaptation procedures based solely on truncation error estimates may over-resolve the wake region, while those including the influence of the adjoint solution will correctly play greater emphasis on decreasing the errors in those cells close to the airfoil which have the greatest influence on the lift and drag.

\section{Unstructured grids}

For unstructured grids, some further analysis is required to obtain good error estimates. Consider a typical discretisation of the 2D or 3D Euler equations using node-based variables, an edge-based data structure, a standard finite volume discretisation of the nonlinear flux terms (which can also be interpreted as a Galerkin finite element discretisation) plus the addition of characteristic smoothing. 
Assuming all of the cells are of bounded aspect ratio, the truncation error at an interior node $j$ can be expressed in the following way as a sum over the set of edges $E_{j}$ coming out of node $j$.

$$
R_{j}=\sum_{k \in E_{j}} h_{k}^{d+1} S_{j k}
$$

Here $h_{k}$ is the length of the edge and there is an implicit assumption that all of the cells are of bounded aspect ratio so that the face associated with the edge has length/area $O\left(h_{k}^{d-1}\right) . \quad S_{j k}$ consists of derivatives of the flow solution; this result comes from the standard integration error from a linear representation of the flux on the face.

Note that since the area/volume of the cell is $O\left(h_{j}^{d}\right)$ where $h_{j}$ is the representative length scale for the cell, the local truncation error normalised by the cell area is $O(h)$. This first order accuracy for the truncation error has led some to believe that the solution accuracy may also be first order. Indeed, if one uses the error representation obtained above,

$$
V^{T} R(u) \equiv \sum_{j} V_{j}^{T} R_{j}
$$

then since the total number of nodes is $O\left(h^{-d}\right)$ where $h$ is the average value for $h_{j}$ over the whole grid, it appears to follow that the error is $O(h)$.

However, numerical evidence suggests that such methods are second order accurate [17]. If one considers a union of neighbouring cells covering a region whose area/volume is $O(1)$, then summing over these cells, the truncation errors for interior fluxes cancel due to conservation. The boundary has $O\left(h^{-d+1}\right)$ faces, each with a truncation error which is $O\left(h^{d+1}\right)$, so the overall truncation error for this aggregation of cells is $O\left(h^{2}\right)$. Giles attempted to refine this argument using a Fourier decomposition of the truncation error and the resulting solution error, but the analysis was not rigorous [18].

To recover the second order accuracy in the error analysis using the adjoint approach requires a simple rearrangement of the error summation. It depends crucially on conservation, so that for an edge $k$ connecting nodes $i$ and $j$, the truncation error associated with the flux along the edge is equal and opposite for the two cells, i.e.

$$
S_{i k}=-S_{j k}
$$

Therefore, the error summation over all cells can be rearranged into a summation over all edges $E$, and all boundary nodes $B$, of the form

$$
\sum_{k \in E} h_{k}^{d+1} \Delta V_{k}^{T} S_{k}+\sum_{j \in B} h_{j}^{d+1} V_{j}^{T} S_{j}
$$

Here $S_{k}$ is the truncation error for the flux along edge $k$ and $\Delta V_{k}$ is the difference in the adjoint solutions at the nodes joined by the edge. Assuming that the 
analytic adjoint solution is differentiable, $\Delta V_{k}$ should be $O\left(h_{k}\right)$, and with the total number of edges being $O\left(h^{-d}\right)$ this means that the first sum is $O\left(h^{2}\right)$. The number of boundary nodes is $O\left(h^{-d+1}\right)$ and so the second sum is also $O\left(h^{2}\right)$. The conclusion is that the overall error in integral quantities, such as lift and drag, is second order even though the local truncation error is first order.

Focusing on the error contribution due to the edges, this is now of the form

$$
\sum_{k \in E} h_{k}^{d+2} T_{k}
$$

where $T_{k}$ is the product of the adjoint solution gradient along the edge and higher order derivatives of the flow solution coming from the Taylor series expansions used to evaluate the flux truncation error.

As with the structured grid error analysis, this error estimate can be used to improve the computed value of the functional. Alternatively, it can be used for grid adaptation through the addition of extra grid nodes, thereby reducing the cell sizes $h_{k}$. The greatest reduction in the error is achieved by introducing additional nodes into the region in which the average magnitude of $h_{k}^{d+2}\left|T_{k}\right|$ is greatest. With repeated refinement, this quantity should eventually become approximately uniform over the grid. The refinement can be continued until the error estimate is smaller than a user-defined tolerance, thereby achieving the goal of minimising the computational cost for a given level of accuracy.

\section{$3 \quad$ Finite element analysis}

To simplify the details of the analysis we restrict consideration to the $2 \mathrm{D}$ or $3 \mathrm{D}$ Poisson equation,

$$
-\nabla^{2} u=f
$$

on a domain $\Omega$ which is a unit square or cube, depending on the dimension, and subject to Dirichlet conditions $u=g$ on the boundary $\partial \Omega$.

\section{Notation and definitions}

We define two inner products, one on the domain,

$$
(u, v) \equiv \int_{\Omega} u v d V
$$

and the other on the boundary,

$$
(u, v)_{\partial \Omega} \equiv \int_{\partial \Omega} u v d A .
$$

It is also convenient to define the following bilinear functional

$$
a(u, v)=(\nabla u, \nabla v) .
$$


The $L_{2}$ norm, $H^{1}$ semi-norm and $H^{1}$ Sobolev norm are given by

$$
\|u\|_{L_{2}}^{2} \equiv(u, u), \quad|u|_{H^{1}}^{2} \equiv a(u, u), \quad\|u\|_{H^{1}}^{2} \equiv(u, u)+a(u, u) .
$$

Semi-norms and Sobolev norms of higher degree are defined similarly.

\section{Standard f.e. analysis}

The standard error analysis for this problem is well-established; see the textbook of Strang \& Fix for full details [1].

The function space $H^{1}(\Omega)$ consists of those functions $u$ for which $\|u\|_{H^{1}}<\infty$. The subspace $H_{0}^{1}(\Omega)$ contains those functions which, in addition, are zero on the boundary $\partial \Omega$, while the subspace $H_{g}^{1}(\Omega)$ has those functions satisfying the Dirichlet b.c. $u=g$.

The weak solution of the problem is given by the function $u \in H_{g}^{1}(\Omega)$ such that

$$
a(u, w)=(f, w), \quad \forall w \in H_{0}^{1}(\Omega)
$$

Now let $S^{h}$ be a finite element subspace of $H^{1}(\Omega)$ consisting of continuous functions which are linear on each triangle (or tetrahedron) of a triangulation $\mathcal{T}^{h}$ of the unit square (or cube), with $h$ being the maximum diameter of any individual cell. The subspace $S_{0}^{h}$ consists of those functions which are zero on the boundary. In addition we will assume that the boundary data $g$ is piecewise linear so that there also exists a subspace $S_{g}^{h}$ with functions satisfying the Dirichlet boundary conditions.

The solution of the finite element problem is the function $u^{h} \in S_{g}^{h}$ such that

$$
a\left(u^{h}, w^{h}\right)=\left(f, w^{h}\right), \quad \forall w^{h} \in S_{0}^{h} .
$$

For any $w^{h} \in S_{0}^{h} \subset H_{0}^{1}(\Omega)$,

$$
a\left(u, w^{h}\right)=\left(f, w^{h}\right),
$$

and hence we obtain the orthogonality property

$$
a\left(u-u^{h}, w^{h}\right)=0, \quad \forall w^{h} \in S_{0}^{h} .
$$

Now, there exist positive constants $C_{1}, C_{2}$ independent of $h$ such that

$$
\begin{aligned}
a(w, w) & \geq C_{1}\|w\|_{H^{1}}^{2}, & & \forall w \in H_{0}^{1}(\Omega), \\
a(v, w) & \leq C_{2}\|v\|_{H^{1}}\|w\|_{H^{1}} & & \forall v, w \in H_{0}^{1}(\Omega) .
\end{aligned}
$$

Hence, using the orthogonality property,

$$
\begin{aligned}
\left\|u-u^{h}\right\|_{H^{1}}^{2} & \leq C_{1}^{-1} a\left(u-u^{h}, u-u^{h}\right) \\
& =C_{1}^{-1} a\left(u-u^{h}, u-w^{h}\right), \\
& \leq C_{1}^{-1} C_{2}\left\|u-u^{h}\right\|_{H^{1}}\left\|u-w^{h}\right\|_{H^{1}},
\end{aligned}
$$


for any $w^{h} \in S_{g}^{h}$. Thus,

$$
\left\|u-u^{h}\right\|_{H^{1}} \leq C_{1}^{-1} C_{2}\left\|u-w^{h}\right\|_{H^{1}} .
$$

At this point $w^{h}$ is chosen to be an interpolant of $u$. Standard results concerning the accuracy of interpolation can then be used to deduce that

$$
\left\|u-u^{h}\right\|_{H^{1}} \leq C_{1}^{-1} C_{2} C_{3} h|u|_{H^{2}},
$$

where $C_{3}$ is another constant independent of $h$.

This proves first order accuracy in the $H^{1}$ norm. To prove second order accuracy in the $L_{2}$ norm requires the Aubin-Nitsche technique of using an adjoint problem. The function $v \in H_{0}^{1}(\Omega)$ is defined by the weak problem

$$
a(v, w)=\left(u-u^{h}, w\right), \quad \forall w \in H_{0}^{1}(\Omega),
$$

and by standard elliptic regularity results there exists a fourth constant $C_{4}$ such that

$$
|v|_{H^{2}} \leq C_{4}\left\|u-u^{h}\right\|_{L_{2}} .
$$

Then, using the orthogonality property again,

$$
\left\|u-u^{h}\right\|_{L_{2}}^{2}=a\left(u-u^{h}, v\right)=a\left(u-u^{h}, v-v^{h}\right), \quad \forall v^{h} \in S_{h}
$$

and so, choosing $v^{h}$ to be an interpolant of $v$,

$$
\begin{aligned}
\left\|u-u^{h}\right\|_{L_{2}}^{2} & \leq C_{2}\left\|u-u^{h}\right\|_{H^{1}}\left\|v-v^{h}\right\|_{H^{1}} \\
& \leq C_{1}^{-2} C_{2}^{3} C_{3}^{2} h^{2}|u|_{H^{2}}|v|_{H^{2}} \\
& \leq C_{1}^{-2} C_{2}^{3} C_{3}^{2} C_{4} h^{2}|u|_{H^{2}}\left\|u-u^{h}\right\|_{L_{2}} \\
\Longrightarrow\left\|u-u^{h}\right\|_{L_{2}} & \leq C_{1}^{-2} C_{2}^{3} C_{3}^{2} C_{4} h^{2}|u|_{H^{2}} .
\end{aligned}
$$

\section{Primal/dual formulations for linear functional}

Suppose now that we are interested in obtaining the value for the following functional which is a combination of inner products over both the domain and the boundary,

$$
I=(u, d)+\left(-\frac{\partial u}{\partial n}, e\right)_{\partial \Omega} .
$$

For an arbitrary function $w \in H_{e}^{1}(\Omega)$, integration by parts yields

$$
(f, w)=a(u, w)+\left(-\frac{\partial u}{\partial n}, e\right)_{\partial \Omega},
$$


where $u$ is still the weak solution of the original (primal) problem. Hence, the problem of determining the value of the linear functional can be expressed in weak form as:

Analytic primal $\mathcal{P}$ : given functions $d, e, f, g$, find $I(d, e, f, g)$ and $u \in H_{g}^{1}(\Omega)$ such that

$$
I+a(u, w)-(f, w)-(u, d)=0, \quad \forall w \in H_{e}^{1}(\Omega) .
$$

The weak formulation of the corresponding dual problem is:

Analytic dual $\mathcal{D}$ : given functions $d, e, f, g$, find $I(d, e, f, g)$ and $v \in H_{e}^{1}(\Omega)$ such that

$$
I+a(w, v)-(f, v)-(w, d)=0, \quad \forall w \in H_{g}^{1}(\Omega) .
$$

The equivalence of the two problems, the fact that they yield the same linear functional $I(d, e, f, g)$, follows immediately from considering $w=v$ in the primal problem, and $w=u$ in the dual.

The finite element approximations to both the primal and dual problems is obtained by replacing $H_{e}^{1}(\Omega)$ and $H_{g}^{1}(\Omega)$ by $S_{e}^{h}$ and $S_{g}^{h}$.

Discrete primal $\mathcal{P}^{h}$ : given functions $d, e, f, g$, find $I^{h}(d, e, f, g)$ and $u^{h} \in S_{g}^{h}$ such that

$$
I^{h}+a\left(u^{h}, w^{h}\right)-\left(f, w^{h}\right)-\left(u^{h}, d\right)=0, \quad \forall w^{h} \in S_{e}^{h} .
$$

Discrete dual $\mathcal{D}^{h}$ : given functions $d, e, f, g$, find $I^{h}(d, e, f, g)$ and $v^{h} \in S_{e}^{h}$ such that

$$
I^{h}+a\left(w^{h}, v^{h}\right)-\left(f, v^{h}\right)-\left(w^{h}, d\right)=0, \quad \forall w^{h} \in S_{g}^{h} .
$$

The equivalence of the linear functionals obtained from these two finite element problems again follows immediately from considering $w^{h}=v^{h}$ in the primal problem, and $w^{h}=u^{h}$ in the dual.

\section{Functional error representation}

Let $u, v$ and $u^{h}$ be the solutions of the problems $\mathcal{P}, \mathcal{D}$ and $\mathcal{P}^{h}$, respectively, and let $w^{h}$ be an arbitrary function in $S_{e}^{h}$. Then, from the definitions of $\mathcal{P}, \mathcal{D}$ and $\mathcal{P}^{h}$, it follows that

$$
\begin{aligned}
a\left(u-u^{h}, v-w^{h}\right)= & a(u, v)-a\left(u^{h}, v\right)-a\left(u, w^{h}\right)+a\left(u^{h}, w^{h}\right) \\
= & -(I-(f, v)-(u, g))+\left(I-(f, v)-\left(u^{h}, g\right)\right) \\
& +\left(I-\left(f, w^{h}\right)-(u, g)\right)-\left(I^{h}-\left(f, w^{h}\right)-\left(u^{h}, g\right)\right) \\
= & I-I^{h} .
\end{aligned}
$$

Similarly, if $u, v$ and $v^{h}$ are the solutions of $\mathcal{P}, \mathcal{D}$ and $\mathcal{D}^{h}$, respectively, and $w^{h} \in S_{g}^{h}$ then

$$
a\left(u-w^{h}, v-v^{h}\right)=I-I^{h} .
$$

Thus we have two different representations of the error $I-I^{h}$ in the finite element approximation of the linear functional. 


\section{A priori error analysis}

The a priori error analysis starts from

$$
I-I^{h}=a\left(u-u^{h}, v-v^{h}\right) .
$$

Using the error bounds from the standard error analysis given before, the error in the functional when using a finite element space of piecewise linear functions is bounded by

$$
\begin{aligned}
\left|I-I^{H}\right| & \leq\left\|u-u^{h}\right\|_{H^{1}}\left\|v-v^{h}\right\|_{H^{1}} \\
& \leq C_{1}^{-2} C_{2}^{2} C_{3}^{2} h^{2}|u|_{H^{2}}|v|_{H^{2}}
\end{aligned}
$$

If one uses a finite element space with polynomials of higher degree such that

$$
\left\|u-u^{h}\right\|_{H^{1}} \leq C_{s} h^{s}|u|_{H^{s+1}}
$$

for some integer $s$ and constant $C_{s}$, then this a priori error estimate becomes

$$
\left|I-I^{H}\right| \leq C_{1}^{-2} C_{2}^{2} C_{s}^{2} h^{2 s}|u|_{H^{s+1}}|v|_{H^{s+1}} .
$$

This shows that the order of accuracy of the finite element approximation of the functional is twice as good as the accuracy of the solution $u^{h}$ in the $H^{1}$ norm. This superconvergence property is due to the fact that the leading order terms in the solution error, $u-u^{h}$, are orthogonal to the smooth functions $d, e$ in the evaluation of the linear functional.

\section{A posteriori error analysis}

The a posteriori error analysis starts from

$$
I-I^{h}=a\left(u-u^{h}, v-w^{h}\right),
$$

where $w^{h}$ will be an interpolation of the dual solution $v$.

Splitting the integral into a sum of integrals over each individual triangle, or tetrahedron, and then integrating by parts yields

$$
I-I^{h}=\sum_{K \in \mathcal{T}^{h}} T_{K}
$$

where

$$
T_{K}=\int_{K}\left(f+\nabla^{2} u^{h}\right)\left(v-w^{h}\right) d V+\frac{1}{2} \int_{\partial K}\left[\frac{\partial u^{h}}{\partial n}\right]\left(v-w^{h}\right) d A,
$$

in which $\left[\frac{\partial u^{h}}{\partial n}\right]$ is the jump in normal derivative across internal faces, and is defined to be zero on faces forming part of the boundary $\partial \Omega$. 
When using piecewise linear finite elements, $\nabla^{2} u^{h}$ is zero within each cell, and $\left[\frac{\partial u^{h}}{\partial n}\right]$ is easily evaluated. The interpolation error $v-w^{h}$ can be estimated from the second derivatives of $v$; these in turn can be estimated using second differences of the dual finite element solution $v^{h}$. In this way, the magnitude of the cell error $T_{K}$ can be accurately estimated.

For optimal grid adaptation, the strategy would be to refine cells for which $\left|T_{K}\right|$ is large until the bound on the error is acceptably small. In the process, $\left|T_{K}\right|$ would become relatively uniform across the whole grid.

\section{Extensions}

The analysis presented here is for a simple p.d.e. (the Poisson equation) on a simple domain (which can be triangulated exactly) and with simple boundary conditions and linear functional (corresponding to functions in the finite element space).

The analysis can be extended to much harder problems. Becker and Rannacher derived the a posteriori error analysis for the incompressible Navier-Stokes equations [8], and Süli, Giles et al developed both a priori and a posteriori analyses for the incompressible Navier-Stokes equations [9]. These analyses assume simple domain boundaries and boundary data. A forthcoming paper by Süli and Giles will show, for the convection/diffusion equation, that smooth curved boundaries and smooth boundary data can be treated in a way which does not destroy the superconvergence property for functionals; the key is an appropriate projection of the boundary geometry and data onto the finite element space.

\section{Some concluding remarks}

This paper has outlined the way in which the solution of an appropriate dual problem can be used to estimate the error in approximating a nonlinear functional in CFD computations. The error estimates can be used either to obtain better approximations to the functional itself, or to drive grid adaptation with the aim of achieving the most accurate answer possible for a given level of computational effort.

The finite volume analysis shows that on unstructured grids discrete conservation is crucial in gaining one order of accuracy relative to the order of the local truncation error. However, the analysis outlined makes the assumption that the gradient of the dual solution is bounded. This may not be true for the Euler equations along the stagnation streamline [16], and so additional analysis may be required.

The a priori finite element error analysis reveals an interesting superconvergence property, showing that the order of accuracy of the approximate linear functional is twice that of the solution itself. The lack of a similar result for 
the finite volume analysis may indicate a significant advantage for finite element methods, but the advantage only appears when using methods which have better than second order accuracy.

\section{Acknowledgments}

I am very grateful to my colleague Endre Süli for his comments on this paper as well as our many discussions regarding error analysis. This research was supported by the UK Engineering and Physical Science Research Council under research grant GR/K91149.

\section{References}

[1] G. Strang and G. Fix. An Analysis of the Finite Element Method. PrenticeHall, 1973.

[2] E. Süli and P. Houston. Finite element methods for hyperbolic problems: a posteriori error analysis and adaptivity. Paper presented as an Invited Lecture at the State of the Art in Numerical Analysis Conference, York, Apr, 1996.

[3] T. Sonar and E. Süli. A dual graph norm refinement indicator for the compressible Euler equations. To appear in Numerische Mathematik, 1997.

[4] E. Süli. A posteriori error analysis and global error control for adaptive finite element approximations of hyperbolic problems. Proceedings of the 16th Biennial Conference in Numerical Analysis, 1996.

[5] I. Babuška and W.C. Rheinboldt. Error estimates for adaptive finite element computations. SIAM Journal of Numerical Analysis, 15(4):736-754, 1978.

[6] I. Babuška and A. Miller. The post-processing approach in the finite element method - Part 1: calculation of displacements, stresses and other higher derivatives of the displacements. International Journal for Numerical Methods in Engineering, 20:1085-1109, 1984.

[7] R. Verfurth. A posteriori error estimation and adaptive mesh-refinement techniques. Journal of Computational and Applied Mathematics, 50:67-83, 1994.

[8] R. Becker and R. Rannacher. Weighted a posteriori error control in finite element methods. Technical report, Universitat Heidelberg, 1994. Preprint No. 96-1. 
[9] M. B. Giles, M. G. Larson, J. M. Levenstam, and E. Süli. Adaptive error control for finite element approximations of the lift and drag in viscous flow. Technical Report NA97/06, Oxford University Computing Laboratory, 1997.

[10] J. Peraire M. Paraschivoiu and A. Patera. A posteriori finite element bounds for linear-functional outputs of elliptic partial differential equations. Symosium on Advances in Computational Mechanics, submitted to Comp. Meth. Appl. Engrg., 1996.

[11] A. Jameson. Aerodynamic design via control theory. Journal of Scientific Computing, 3:233-260, 1988.

[12] A. Jameson, N.A. Pierce, and L. Martinelli. Optimum aerodynamic design using the Navier-Stokes equations. AIAA Paper 97-0101, 1997.

[13] O. Baysal and M.E. Eleshaky. Aerodynamic sensitivity analysis methods for the compressible Euler equations. Journal of Fluids Engineering, 113:681688, 1991.

[14] J. Elliott and J. Peraire. Practical 3D aerodynamic design and optimization using unstructured grids. AIAA Paper 96-4122-CP, 1996. Proceedings of 6th AIAA/NASA/ISSMO Symposium on Multidisciplinary Analysis and Optimization.

[15] W.K. Anderson and V. Venkatakrishnan. Aerodynamic design optimization on unstructured grids with a continuous adjoint formulation. AIAA Paper 97-0643, 1997.

[16] M.B. Giles and N. A. Pierce. Adjoint equations in CFD: duality, boundary conditions and solution behaviour. AIAA Paper 97-1850, 1997.

[17] D.R. Lindquist and M.B. Giles. A comparison of numerical schemes on triangular and quadrilateral meshes. In D.L. Dwoyer, M.Y. Hussaini, and R.G. Voigt, editors, Proceedings of the 11th International Conference on Numerical Methods in Fluid Dynamics, volume 323 of Lecture Notes in Physics, pages 369-373, Berlin, 1989. Springer-Verlag.

[18] M.B. Giles. Accuracy of node based solutions on irregular meshes. In D.L. Dwoyer, M.Y. Hussaini, and R.G. Voigt, editors, Proceedings of the 11th International Conference on Numerical Methods in Fluid Dynamics, volume 323 of Lecture Notes in Physics, pages 273-277. Springer-Verlag, 1989. Berlin. 